\title{
Selective hydrophilic modification of Parylene $C$ films: a new approach to cell micro-patterning for synthetic biology applications
}

\author{
Trantidou $\mathbf{T}^{1^{*}}$, Rao $\mathrm{C}^{2,3}$, Barrett $\mathrm{H}^{2}$, Camelliti $\mathrm{P}^{2}$, Pinto $\mathrm{K}^{2}$, Yacoub $\mathbf{M H}^{2}$, Athanasiou $\mathrm{T}^{3}$, \\ Toumazou $\mathrm{C}^{1}$, Terracciano $\mathrm{CM}^{2}$ and Prodromakis $\mathbf{T}^{1,4}$ \\ ${ }^{1}$ Centre for Bio-Inspired Technology, Institute of Biomedical Engineering, Imperial College London, \\ SW7 2BT, UK \\ ${ }^{2}$ National Heart and Lung Institute, Imperial College London, W12 0NN, London, UK \\ ${ }^{3}$ Department of Surgery and Cancer, Imperial College London, London, UK \\ ${ }^{4}$ Nano Research Group, Department of Electronics and Computer Science, University of \\ Southampton, Southampton, SO17 1BJ, UK
}

\begin{abstract}
We demonstrate a simple, accurate and versatile method to manipulate Parylene $\mathrm{C}$, a material widely known for its high biocompatibility, and transform it to a substrate that can effectively control the cellular microenvironment and consequently affect the morphology and function of the cells in vitro. The Parylene C scaffolds are fabricated by selectively increasing the material's surface water affinity through lithography and oxygen plasma treatment, providing free bonds for attachment of hydrophilic biomolecules. The micro-engineered constructs were tested as culture scaffolds for rat ventricular fibroblasts and neonatal myocytes (NRVM), towards modeling the unique anisotropic architecture of native cardiac tissue. The scaffolds induced the patterning of extracellular matrix compounds and therefore of the cells, which demonstrated substantial alignment compared to typical unstructured cultures. $\mathrm{Ca}^{2+}$ cycling properties of the NRVM measured at rates of stimulation $0.5-2 \mathrm{~Hz}$ were significantly modified with a shorter time to peak and time to $90 \%$ decay, and a larger fluorescence amplitude $(p<0.001)$. The proposed technique is compatible with standard cell culturing protocols and exhibits long-term pattern durability. Moreover, it allows the integration of monitoring modalities into the micro-engineered substrates for a comprehensive interrogation of physiological parameters.
\end{abstract}

\footnotetext{
${ }^{*}$ Corresponding author. Email: tatiana.trantidou09@imperial.ac.uk
} 
Keywords: Parylene C, hydrophobic, hydrophilic, patterning, cardiac tissue engineering, calcium cycling

\section{Introduction}

Poly(chloro-para-xylylene) or Parylene $\mathrm{C}$ is a material with a long history of use in the field of medical industry as a biocompatible encapsulant of implantable biomedical devices, such as neuroprosthetic devices [1], cardiovascular implants [2], stents and catheters [3]. Besides, Parylene C has been widely employed as an effective packaging approach due to its chemical inertness, nondegradability and high encapsulation quality in order to facilitate the integration of biosensor interfaces and electronic detection circuitry $[4,5]$. The material's excellent encapsulation performance has been primarily attributed to Parylene's inherent hydrophobic (site-free) nature, pinhole free structure above $1 \mu \mathrm{m}$ [6] and low water vapor transmission rate [7], enabling the material to act as an electrolytebarrier.

In addition to its numerous encapsulation applications, Parylene $C$ has recently gained particular interest as a structure material for flexible electronics due to its excellent mechanical and thermal properties that enable the integration of monitoring modalities into flexible implementations. Such examples are the flexible implantable Parylene C sensor performing intraocular pressure measurements in glaucoma patients [8], as well as the Parylene-based multi-electrode array (MEA) for neural stimulation and recording [9]. The material's mechanical robustness derives from its high Young's modulus (1 GPa for 2-11 $\mu \mathrm{m}$ ), which makes the material highly suitable for producing stable and reusable microfluidic devices [10] and stencils for cell and protein patterning [11, 12].

The naturally occurring hydrophobic nature of Parylene $\mathrm{C}$ certainly has rendered the material extremely popular for encapsulation purposes, but prevented its use in applications that involve a direct contact with biological material. Many research efforts have been realized to alter the hydrophobic properties of Parylene $\mathrm{C}$ with particular focus on cell culturing. Oxygen plasma treatment has been previously employed to effectively modify the surface properties of such films in order to make the material suitable as a cell culture substrate [13, 14]. Selective patterning of hydrophilic and superhydrophobic areas on a Parylene C 3-D microstructure was achieved through employing two distinct plasma treatments, $\mathrm{O}_{2}$ and $\mathrm{SF}_{6}$ respectively, with applications in droplet-based microfluidics [15]. Other studies exploited Parylene $C$ as a substrate for patterning neural cells and 
demonstrated that cell adhesion on Parylene is promoted by hydrophobic absorption of the serum proteins, which in fact is reduced when Parylene is oxidized through UV irradiation [16].

The need to model the complex interactions between cellular communities in living tissues and produce representative in vitro disease and pharmacological models remains one of the greatest challenges in synthetic biology. Microcontact stamping/printing achieves the selective deposition on a substrate of attachment factors, such as fibronectin [17] and laminin [18], using polydimethylsiloxane (PDMS) stamps. Patterning cells inside microfluidic channels allows the selective transfer of the extracellular matrix proteins and cells to a substrate by applying the solution at the entry of the microchannel and performing suction at the last end [19]. The aforementioned techniques, however, involve complicated steps which are difficult to perform and lack reproducibility [20]. Moreover, these methods can be difficult to integrate with biosensors, such as MEAs, where alignment with the engineered construct is critical. Structured PDMS fabricated through mold casting on a master silicon wafer has also been used as micro-grooved scaffolds to promote structuring of induced pluripotent stem cells-derived cardiac myocytes (iPSC-CM) [21], however, this method does not facilitate the integration of monitoring modalities into the structured cultures due to the weak mechanical and structural properties of the material [22]. Selective molecular assembly patterning combines photolithographic procedures and the self-assembly of organic molecules in order to engineer one cell adhesive and one cell repulsive substrate [23], but it relies on the careful selection of the appropriate chemistry in order to assemble organic molecules in a two-step procedure without disrupting previously assembled molecules. Patterning of mouse myoblasts cells on a hydrophobic dielectric surface inside an engineered microfluidic device was achieved through controlling electrosurface phenomena with embedded microelectrodes [24]. These electrically programmable assembly patterning techniques require very high voltages $(60 \mathrm{~V})$ to bias the desired microelectrodes in order to achieve adequate hydrophilicity of the dielectric substrate. Microfabricated polymer stencils is another method to engineer the cellular microenvironment by serving as selective physical barriers that are peeled-off after seeding. Due to its high Young's modulus, Parylene $\mathrm{C}$ has been widely used to pattern proteins [11, 12] and cells [12]. Nonetheless, this technique involves a very delicate procedure to peel-off the Parylene stencil intact, which often results in partial destruction of the polymer membrane and residual pieces on the supporting surface especially when thin $(\sim 1 \mu \mathrm{m})$ stencils are used. 
In a recent research study we investigated the effect of oxygen plasma treatment on Parylene $\mathrm{C}$ films, extrapolating correlations between treatment parameters (power intensity and exposure time) and the extent of the induced hydrophilicity. We have also demonstrated how to selectively modify the surface hydrophobicity of Parylene using lithography and oxygen plasma treatment [25]. Here, we report a method for manipulating Parylene $C$ substrates to produce scaffolds that construct 2-D cellular microenvironments and control their morphology and physiology. We demonstrate the versatility of the proposed method through application with two distinct types of attachment factors and cardiac cells (myocytes and fibroblasts) and we study its impact on the cellular structure and function. The approach presented here highlights a low-cost, simple and easily implementable technique that is compatible with standard cell culturing protocols and yields accurate, reproducible long-term results.

\section{Materials and methods}

\subsection{Fabrication of micro-engineered Parylene $C$ constructs}

The fabrication process is outlined in figure 1. Briefly, standard glass coverslips $(\varnothing 25 \mathrm{~mm})$ were thoroughly degreased in acetone (ACE), isopropanol (IPA) and deionized (DI) water, and dehydrated for $60 \mathrm{~s}$ at $90{ }^{\circ} \mathrm{C}$. Parylene $\mathrm{C}$ films were deposited by Chemical Vapor Deposition, using a commercially available coater (PDS2010) by vaporizing $\left(150{ }^{\circ} \mathrm{C}\right)$ and then pyrolizing Parylene $\mathrm{C}$ dimer $\left(690^{\circ} \mathrm{C}\right)$. Three runs were carried out, facilitating three different thicknesses, and the samples were accordingly divided into three groups; (a) $1 \mu \mathrm{m}$ (group 1), (b) $7 \mu \mathrm{m}$ (group 2) and (c) $10 \mu \mathrm{m}$ (group 3). In all cases, the film thickness was determined through calibration samples where Parylene C was selectively removed to create a step that was measured by a Veeco Dektak Stylus profiler. Hexamethyldisilazane (HMDS) was subsequently spin-coated on the Parylene C coated coverslips, succeeded by a $1.4 \mu \mathrm{m}$ thick positive photoresist (AZ5214). The samples were then soft baked on a hotplate at $90{ }^{\circ} \mathrm{C}$ for $60 \mathrm{~s}$, selectively exposed to UV light for $60 \mathrm{~s}$ through a chromeplated glass mask, consisting of transparent areas with parallel lines. The samples were subsequently developed in $\mathrm{AZ400K}-\mathrm{H}_{2} \mathrm{O}(1: 4)$ to remove the exposed photoresist. Oxygen plasma treatment was performed using an ultra high purity plasma etcher (Nano UHP) at a working pressure of 0.8 mbar. Before inserted into the plasma etcher, samples were prebaked on a hotplate at $110{ }^{\circ} \mathrm{C}$ for $60 \mathrm{~s}$ for hardening the protective photoresist mask. Selective hydrophilic patterning was achieved 
through oxygen plasma treatment of the Parylene C films. After plasma treatment, the remaining photoresist was removed through immersing the constructs into ACE, IPA and DI water. The resulting surface layout of the Parylene $C$ substrates had parallel $10 \mu \mathrm{m}$ wide hydrophobichydrophilic lines. The dimensions were determined based on previous studies with NRVM suggesting that lines of this width most effectively aligned and improved the $\mathrm{Ca}^{2+}$ cycling properties of cardiomyocytes [21]. The power intensity of the $\mathrm{O}_{2}$ plasma treatment defines the etching rate according to our previous findings [25]. For culturing NRVM, two types of selectively hydrophilic Parylene $C$ films were fabricated; in the first case, $1 \mu \mathrm{m}$ thick Parylene films were treated at $400 \mathrm{~W}$ for $15 \mathrm{~min}$. The exposed Parylene $\mathrm{C}$ areas were completely etched to reveal the hydrophilic glass substrate underneath (glass/hydrophobic $-\mathrm{G} / \mathrm{H}$ ). In the second case, the $7 \mu \mathrm{m}$ thick Parylene $\mathrm{C}$ films received oxygen plasma treatment at $50 \mathrm{~W}$ for $1 \mathrm{~min}$. Selective hydrophilic modification of the film surface was accomplished without significant etching (hydrophilic/hydrophobic $-\mathrm{H} / \mathrm{H}$ ). Parylene $\mathrm{C}$ constructs $\left(7 \mu \mathrm{m}\right.$ thick) that were conformally exposed to $\mathrm{O}_{2}$ plasma (50 $\mathrm{W}-1 \mathrm{~min}$ ) comprised the control (non-engineered) group. For culturing fibroblasts, Parylene C samples from group 3 were treated at $400 \mathrm{~W}$ for $5 \mathrm{~min}$, resulting in hydrophobic-hydrophilic grooves of $0.5 \mu \mathrm{m}$ in depth. Parylene C constructs of same thickness were conformally exposed to $\mathrm{O}_{2}$ plasma ( $400 \mathrm{~W}-5 \mathrm{~min}$ ) to serve as the control (non-engineered) groups. Throughout this paper, we refer to the micro-engineered constructs and the cells cultured on these as "patterned" and "structured" respectively, whereas the control (non-engineered) substrates and the corresponding cell cultures are mentioned as "unpatterned" and "unstructured" respectively.

\subsection{Cardiomyocytes isolation and culture}

NRVM were isolated from Sprague-Dawley rats 2 days after birth. All experiments were conducted in accordance with Home Office regulations detailed in the Animals (Scientific Procedures) Act 1986. Two isolations were performed for experiments with the $\mathrm{G} / \mathrm{H}$ and $\mathrm{H} / \mathrm{H}$ constructs and four isolations for experiments with the control constructs. The isolation technique has been described previously [26]. Briefly neonatal ventricles were excised and enzymatically digested. These digestions were centrifuged to produce a cell pellet which was resuspended in $25 \mathrm{ml}$ Complete Medium (Dulbecco's Modified Eagle Medium, DMEM) containing 4.5g/L glucose (Gibco), 25\% medium 199 (Sigma), penicillin-streptomycin (Sigma), 15\% horse serum (Sigma), 7\% fetal bovine serum (FBS, Sigma) and 
3.4\% HEPES (Sigma)). The solution was pre-plated for $1 \mathrm{~h}$ to remove fibroblasts. The cells were then swept from the plate and counted on a light microscope using a haemocytometer. Prior to seeding, all constructs were sterilized by submersion in $100 \%$ ethanol for $1 \mathrm{~h}$. The samples were subsequently washed with sterile phosphate buffered saline (PBS) (Oxoid), coated with $5 \mu \mathrm{g} / \mathrm{ml}$ fibronectin (Sigma) and stored in an incubator $\left(37^{\circ} \mathrm{C}, 5 \% \mathrm{CO}_{2}\right)$ for $20 \mathrm{~h} .2 \times 10^{6}$ cells were seeded at identical densities on each construct (patterned and unpatterned). All seeded constructs were then incubated $\left(37^{\circ} \mathrm{C}, 5 \% \mathrm{CO}_{2}\right)$. Complete medium was changed every other day. All experiments were performed at day 4 post-seeding.

\subsection{Fibroblasts isolation and culture}

Ventricular fibroblasts were prepared from adult Sprague-Dawley rats (two isolations). All experiments were conducted in accordance with Home Office regulations detailed in the Animals (Scientific Procedures) Act 1986. The isolation technique has been described previously [27]. Briefly, ventricles were excised and enzymatically digested. The cell suspension was spun at $620 \mathrm{~g}$ for 4 min to collect the fibroblasts. The fibroblasts were cultured in $25 \mathrm{ml}$ DMEM (Sigma) containing $10 \%$ FBS (Sigma). Prior to seeding, all constructs were sterilized by double submersion in $70 \%$ ethanol for 10 min. The samples were left to dry in a sterile environment and subsequently washed twice with PBS (Oxoid) and sterile deionized water. After drying out, the constructs were coated with a solution consisting of $5 \mathrm{mg}$ type IV human placenta collagen (Sigma) diluted in $22 \mathrm{ml}$ of Hank's Balanced Salts (Sigma) and $3 \mathrm{ml}$ glacial acid, then washed thrice with sterile DI water and stored in an incubator $\left(37^{\circ} \mathrm{C}, 5 \% \mathrm{CO}_{2}\right) .5 \times 10^{4}$ cells were seeded on each construct. All seeded constructs were then incubated $\left(37{ }^{\circ} \mathrm{C}, 5 \% \mathrm{CO}_{2}\right)$. Complete medium was changed 18 hours after seeding. Immunofluorescence experiments were performed at days 3-4 post-seeding.

\subsection{Immunofluorescence}

To determine cell alignment NVRM cytoskeletal myosin heavy chains were labeled using fluorescent Anti-heavy chain cardiac myosin antibody (Abcam), while nuclear deoxyribonucleic acid was labeled with fluorescent 4',6-diamidino-2-phenylindole (DAPI) (Invitrogen). The plasma membranes of the fibroblasts were labeled by incubating the cells with $15 \mathrm{mM}$ di-8-ANEPPS (Invitrogen) for $5 \mathrm{~min}$, while their nuclear deoxyribonucleic acid was labeled with Hoechst 33258 (H258, Invitrogen). Images were 
taken using an Upright Zeiss confocal microscope. Alignment was assessed through an automated process using a dedicated image processing software tool (ImageJ, NIH, Baltimore).

\subsection{Measurement of $\mathrm{Ca}^{2+}$ transients}

Calcium transients were studied by loading the NRVM on the constructs with fluo-4-AM, a nonratiometric $\mathrm{Ca}^{2+}$ sensitive fluorescent dye. NRVM were loaded with $1 \mathrm{ml}$ DMEM, $10 \mu \mathrm{M}$ fluo-4-AM (Invitrogen) with $0.2 \%$ pluronic acid (Invitrogen) and $4 \mu \mathrm{M}$ probenacid (Invitrogen) for 30 minutes at $37^{\circ} \mathrm{C}$ in a humidified atmosphere with $5 \% \mathrm{CO}_{2}$. They were then de-esterified in $1 \mathrm{ml}$ DMEM with $2 \%$ FBS and $4 \mu \mathrm{M}$ probenacid for a further 30 minutes at $37^{\circ} \mathrm{C}$ in a humidified atmosphere with $5 \% \mathrm{CO}_{2}$. The calcium transients collected from single cells were studied using line scanning on an Upright Zeiss confocal microscope using $488 \mathrm{~nm}$ excitation wavelength at $40 \mathrm{x}$ magnification. Fluorescence emissions were collected through a long pass $505 \mathrm{~nm}$ filter. NRVM were field-stimulated at $0.5,1$ and $2 \mathrm{~Hz}$ to induce rhythmic depolarization. Line scans were converted into calcium transients using ImageJ (NIH, Baltimore) and analyzed blindly in Clampfit 10.0 (Molecular Devices) to calculate amplitude (f/f0), time to peak, time to $50 \%$ and time to $90 \%$ decline.

\subsection{Statistical analysis}

Statistical analysis of the alignment and calcium properties of cardiomyocytes was performed using a 1-way ANOVA Kruskal Wallis test with a confidence interval of $95 \%$. Statistical analysis of the alignment of fibroblasts was performed using an unpaired $t$ test. Data are expressed as mean \pm SEM unless specified otherwise. Throughout this study, $n$ represents the number of cells (myocytes or fibroblasts). In the figures, ${ }^{*}$ indicates $p<0.05 ;{ }^{* *} p<0.01$; and ${ }^{* * *} p<0.001$. The analysis was performed using Prism 4 software (GraphPad software Inc).

\section{Results}

\subsection{Evaluation of Parylene $C$ micro-engineered constructs}

The fabrication process is outlined in figure 1a. The evaluation of this method was initially performed by coating the engineered and non-engineered (hydrophobic) Parylene $C$ constructs with green fluorescent fibronectin (HiLyte 488, AnaSpec Inc.). Figures $1 \mathrm{~b}$ and 1c illustrate the corresponding results obtained with a fluorescent microscope, indicating that fibronectin is absorbed by the hydrophilic areas only. Profilometer scan profiles acquired from the three distinct groups of micro- 
engineered constructs (1, 7 and $10 \mu \mathrm{m}$ thick Parylene $\mathrm{C}$ films) are presented in figure $1 \mathrm{~d}$. As a consequence, the NRVM cells that are subsequently seeded on these constructs self-align on the areas where the attachment factors exist. Figure 2 presents the bright-field and immunofluorescent pictures of the NRVM seeded on non-engineered Parylene constructs with their surface being treated hydrophilic and micro-engineered $(H / H$ and $\mathrm{G} / \mathrm{H})$ Parylene constructs 1 week after production, demonstrating the alignment of the cells in the direction of the construct lines. NRVM attached and aligned similarly when seeded on the micro-engineered constructs 4 weeks after production, demonstrating the long-term usability of the proposed method (Appendix).

Fibroblasts seeded on collagen coated micro-engineered constructs attached and aligned in the direction of the construct (Figures 3a-3b). Similarly to fibronectin, collagen binds only on the hydrophilic lines. The pattern in the collagen coated constructs was visible during confocal analysis in areas without cells and is shown in scale in figure $3 b$ (inset) for comparison. It is worth mentioning that in confluent cultures where the cell density is high, cells tend to bridge across the hydrophobic lines, nevertheless, they orientate parallel to the direction of lines. This phenomenon is more profound in constructs with shallower groves (Figure 2e) and with cells that have a bigger size relatively to the geometry of the layout, such as the adult rat fibroblasts (Figure 3b).

\subsection{Cell alignment}

The micro-engineered Parylene C substrates significantly improved NRVM alignment compared to the unpatterned (control) constructs. The results demonstrate that between the two micro-engineered types of constructs, the modulus of angle of cell from mean axis is smaller for cells seeded on the grooved G/H substrates, indicating a further reduction in modulus by $15.18 \%$ (Figure $2 \mathrm{~g}$ ). Similarly, fibroblasts seeded on the micro-engineered Parylene $C$ constructs demonstrated considerable $(p$ $<0.001$ ) alignment to the direction of the lines compared to unstructured cultures (Figure $3 c$ ). To evaluate the elongation of the cells, the ratio of the major to the minor axis of the cell was assessed (analyzing the cellular nucleus as an eclipse) (Figure 3d). Fibroblasts' nuclei were significantly more elongated on the $\mathrm{H} / \mathrm{H}$ micro-engineered constructs $(\mathrm{p}<0.01)$ compared to typical cell cultures.

\subsection{Calcium cycling}

NRVM cultured on the engineered substrates had a larger $\mathrm{Ca}^{2+}$ transient amplitude $\left(f / f_{0}\right)$ at all 
stimulation frequencies $(p<0.001)$ compared to the ones cultured on the non-engineered constructs. Time to transient peak $(T p)$ was shorter only for cells on the $\mathrm{H} / \mathrm{H}$ construct at all stimulation frequencies ( $p<0.01$ at $0.5 \mathrm{~Hz}, p<0.05$ at $1 \mathrm{~Hz}$ and $p<0.001$ at $2 \mathrm{~Hz}$ ), whereas cells on $\mathrm{G} / \mathrm{H}$ substrates did not present any change in terms of time to transient peak. Time to $50 \%$ transient decay $(T 50)$ was significantly $(p<0.001)$ reduced for both types of micro-engineered constructs only when the culture was stimulated at $2 \mathrm{~Hz}$. Finally, time to $90 \%$ transient decay (T90) was considerably reduced for both types of structured cultures at all stimulation frequencies $(p<0.001$ at $0.5 \mathrm{~Hz}, \mathrm{p}<0.05$ for $\mathrm{H} / \mathrm{H}$ and $\mathrm{p}<0.001$ for $\mathrm{G} / \mathrm{H}$ at $1 \mathrm{~Hz}$ and $\mathrm{p}<0.001$ at $2 \mathrm{~Hz}$ ).

There was no statistically significant difference in the transient amplitude $\left(f / f_{0}\right)$, time to $50 \%$ and time to $90 \%$ decay between cells cultured on $\mathrm{H} / \mathrm{H}$ and $\mathrm{G} / \mathrm{H}$ constructs. However, structured cultures on the $\mathrm{H} / \mathrm{H}$ constructs had a shorter time to transient peak compared to the $\mathrm{G} / \mathrm{H}$ substrates at all stimulation frequencies $(p<0.001)$ (Figure 4).

Figure 5 shows the frequency dependency of the $\mathrm{Ca}^{2+}$ transient parameters of NRVM cultured on unpatterned and patterned Parylene C constructs. All cultures showed the expected negative relationship for $\mathrm{Ca}^{2+}$ transient amplitude. However, as for more mature myocardium, there was a faster decline of the transients at high frequencies in the patterned constructs, which was not observed in unpatterned constructs. In particular, the $\mathrm{Ca}^{2+}$ transient amplitude and decline (T50 and T90) of the cells on the $\mathrm{H} / \mathrm{H}$ and $\mathrm{G} / \mathrm{H}$ construct were significantly $(p<0.001)$ reduced as the stimulation frequency increases from 0.5 to $2 \mathrm{~Hz}$.

\section{Discussion}

In this paper, we demonstrate a simple and reliable method to fabricate Parylene C scaffolds that induce the self-alignment of cells and proteins. The Parylene $\mathrm{C}$ scaffolds are fabricated by selectively modifying the surface water affinity of the Parylene through standard lithography and oxygen plasma treatment creating hydrophilic groups able to bind other hydrophilic molecules. The degree of hydrophilicity as well as the configuration can be accurately tuned by defining the oxygen plasma treatment parameters (power intensity and exposure time). In order to highlight the versatility of the proposed method, we employed a range of film thicknesses $(1,7$ and $10 \mu \mathrm{m})$ and oxygen plasma conditions (50 W/1 $\mathrm{min}, 400 \mathrm{~W} / 15 \mathrm{~min}, 400 \mathrm{~W} / 5 \mathrm{~min}$ ) and produced two distinct groups of hydrophobic/hydrophilic constructs: (a) non-grooved $(\mathrm{H} / \mathrm{H})$ and (b) grooved (1 $\mu \mathrm{m}$ and $0.5 \mu \mathrm{m}$ deep) 
constructs. The selective exposure of the Parylene $\mathrm{C}$ surface to $\mathrm{O}_{2}$ plasma locally changes the inherent hydrophobic (site-free) nature of the polymer, resulting in hydrophilic areas that are able to bind the attachment factors, such as fibronectin or collagen. Previous studies have attributed Parylene's induced hydrophilicity to the formation of free carboxyl groups $(C=O)$ on the polymer's surface $[28,29]$. The active sites are present even 4 weeks after production (see Appendix), which strongly demonstrates the long-term applicability of the proposed method.

The micro-engineered constructs were tested with NRVM cells and rat ventricular fibroblasts in order to investigate any improvement to the cellular morphology and function towards a more representative model of native cardiac tissue. We concentrated on two configurations for patterning NRVM; a) a construct without grooves $(50 \mathrm{~W} / 1 \mathrm{~min})$ to indicate that the alignment is a result of the surface chemistry and not the surface geometry, and b) a construct with $1 \mu \mathrm{m}$-deep grooves $(400 \mathrm{~W} / 15 \mathrm{~min})$ to indicate that the alignment is a result of combined surface geometry and surface chemistry. Finally, we showed an intermediate state with $0.5 \mu \mathrm{m}$-deep groves, where surface chemistry and geometry $(400 \mathrm{~W} / 5 \mathrm{~min})$ both contribute to the patterning of cardiac fibroblasts. The micro-patterned substrates significantly promoted alignment in both cell types, indicating the wide applicability of the proposed method. In particular NRVM alignment was significantly $(p<0.001)$ promoted in both types of patterned substrates $(\mathrm{H} / \mathrm{H}$ and $\mathrm{G} / \mathrm{H})$ compared to cells on non-engineered constructs that are conformally hydrophilic. Comparing the two patterned constructs, alignment was greater in the grooved $(\mathrm{G} / \mathrm{H})$ substrates. Although alignment here is primarily a result of the surface chemistry of the Parylene constructs, the geometry of the support substrate would also have an impact, as we have previously demonstrated using micro-grooved PDMS scaffolds that are conformally hydrophilic [21]. Other studies employing NRVM [17] as well as rat dermal fibroblasts [30] have also indicated that deeper grooves induce better cell alignment. Based on our previous findings and the work undertaken here, it is conclusive that alignment is a result of both the surface chemistry and the surface geometry of the Parylene C substrate. The degree of the induced alignment is directly related to the oxygen plasma treatment parameters (power intensity and treatment time) which in turn define the etching rate of the Parylene surface [25].

Parylene $\mathrm{C}$ micro-engineered substrates significantly changed the $\mathrm{Ca}^{2+}$ cycling properties of NRVM. Cells on micro-engineered constructs also had a larger $\mathrm{Ca}^{2+}$ amplitude and faster decline when stimulated at all frequencies of field stimulation. The change in amplitude in NRVM cells is likely to 
be attributed to changes in the sarcoplasmic reticulum (SR) function, given the relative importance of $\mathrm{SR} \mathrm{Ca}^{2+}$ to calcium-induced-calcium-release (CICR) in rats and mice [31], however we cannot completely exclude a contribution from extra-sarcolemma $\mathrm{Ca}^{2+}$. Increased sarcoplasmic reticular calcium load has been shown to increase ryanodine receptor (RyR) open probability [32]. Because of this effect an increase in SR calcium load would increase calcium release so increasing the amplitude. The time to peak calcium transient would also increase, if there was not a corresponding increase in RyR function. Increased contribution of $\mathrm{SR} \mathrm{Ca}^{2+}$ in the patterned constructs is also suggested by the negative force-frequency relationship only observed in structured cultures as at higher frequencies the $\mathrm{SR} \mathrm{Ca}^{2+}$ content is reduced in rats and mice cells, resulting in reduced amplitude [31].

The ability to shorten $\mathrm{Ca}^{2+}$ extrusion at higher frequencies, as the consequence of a shorter action potential and other adaptations of the excitation-contraction coupling machinery [33], are fundamental properties of mature myocardium. This phenomenon was clearly observed in both Parylene C micro-engineered substrates but not in the unstructured cultures. A reduction in sarco/endoplasmic reticulum $\mathrm{Ca}^{2+}$-ATPase (SERCA) function has been shown to reduce the rate constant of calcium transient decay [34]. It would be expected that a change in SERCA function, improving reuptake into the sarcoplasmic reticulum, would alter decay time at $50 \%$. As the reduction was seen only significant for the $90 \%$ decay time changes in other calcium handling mechanisms such as the sodium-calcium exchanger, mitochondrial calcium handling or the sarcolemmal calcium ATPase may also be implicated.

Interestingly, $\mathrm{Ca}^{2+}$ transient increased faster in NRVM after stimulation only for the $\mathrm{H} / \mathrm{H}$ construct at all three frequencies. In the context of a larger $\mathrm{Ca}^{2+}$, transient amplitude this probably also reflects a corresponding improvement in RyR function. This observation strongly suggests that the greater degree of alignment induced by the deeper grooves in the $\mathrm{G} / \mathrm{H}$ constructs, which has previously been described [21], is not the only factor responsible for the observed modulation in cellular function.

Several culture techniques which promote cellular alignment have been show to improve $\mathrm{Ca}^{2+}$ cycling in NRVM. Microgrooved substrates have been showed to increase the speed of $\mathrm{Ca}^{2+}$ cycling [20], and to elevate diastolic and systolic calcium [35]. Aligned collagen constructs increase voltagegated calcium currents in NRVM [36]. NRVM cultured on microcontact printed lines have lower 
diastolic calcium, larger calcium transient amplitude and a trend towards faster calcium removal [37]. The changes in calcium handling reported with NRVM culture on microcontact printed constructs are particularly interesting, as the findings are similar to our own results, suggesting that these constructs like the $\mathrm{H} / \mathrm{H}$ plasma-treated constructs probably also enforce structure on NRVM without geometric constraints.

Despite the evidence showing an association between cellular alignment and improved $\mathrm{Ca}^{2+}$ cycling in NRVM it is not clear why this should occur. Cytoskeletal reorganisation in response to external cues has previously been demonstrated in several studies [38-40]. It may be that this reorganization promotes better SR organization which influences $\mathrm{Ca}^{2+}$ cycling, or that $\mathrm{Ca}^{2+}$ cycling is modified by independent mechanisms. Further research needs to be undertaken to elucidate this.

The main advantages of the proposed fabrication technique lie in its simplicity, versatility, scalability and precision. Simplicity is achieved both in terms of the fabrication process, but mainly because the application in cell culturing does not require any complex equipment and/or procedures, since extracellular matrix compounds are simply applied to the fabricated constructs, significantly facilitating the biologically-oriented user. Versatility and scalability are achieved using multiple layout geometries tailored to a specific biological application and enabling microscale patterning resolution down to $1 \mu \mathrm{m}$. Finally, precision is accomplished using conventional well optimized micro-fabrication processes that allow the reproducibility of the patterned layout with minimum deviation. This microfabrication compatible approach also facilitates the introduction of monitoring features into the patterned substrates and vice versa. An indicative example would be the transfer of the patterning technology to commercial tools performing electrical monitoring, such as commercial 2-D MEAs, in order to study the cellular electrophysiology.

\section{Conclusions}

This study presents a simple, cost-effective and reliable method to produce 2-D Parylene C constructs for self-alignment applications of cells and proteins. We demonstrated that the technique can deliver promising cardiac cell scaffolds in order to model the unique anisotropic architecture and function of native cardiac tissue. Clearly, this approach is not limited to the cell types demonstrated here and could be optimized according to specific application requirements for a variety of cell types and biomolecules. The proposed technique is compatible with standard micro-fabrication processes, 
allowing the integration of monitoring features in order to study action potential propagation of structured cultures. Furthermore, since Parylene's excellent mechanical properties facilitate the production of free-standing ultrathin (down to $1 \mu \mathrm{m}$ ) flexible films, this method could potentially find interesting applications in stretch-induced maturation systems to investigate the effect on cardiomyocyte organization and structure.

\section{Acknowledgments}

The authors would like to acknowledge the financial support of the A.G. Leventis foundation and the Wellcome Trust. We also thank James Cartledge for isolating the fibroblasts.

\section{References}

[1] Loeb GE, Walker AE, Uematsu S, Konigsmark BW. Histological reaction to various conductive and dielectric films chronically implanted in the subdural space. Journal of Biomedical Material Research 1977;11(2):195-210.

[2] Schmidt EM, McIntosh JS, Bak MJ. Long-term implants of parylene-C coated microelectrodes. Medical and Biological Engineering and Computing 1988;26(1):96-101.

[3] Wolgemuth L. Crystal-clear coating covers components. Medical Design. 2006;6:48-51.

[4] Prodromakis T, Michelakis K, Zoumpoulidis T, Dekker R, Toumazou C. Biocompatible encapsulation of CMOS based chemical sensors. IEEE Sensors Conference 2009:791-794.

[5] Li L, Mason AJ. Post-CMOS Parylene Packaging for On-chip Biosensor Arrays. IEEE Sensors Conference 2010:1613-1616.

[6] Trantidou T, Payne D, Tsiligkiridis V, Chang Y-C, Toumazou C, Prodromakis T. The dual role of Parylene $\mathrm{C}$ in chemical sensing: Acting as an encapsulant and as a sensing membrane for $\mathrm{pH}$ monitoring applications. Sensor and Actuators B 2013;186:1-8.

[7] Xie X, Rieth L, Caldwell R, Diwekar M, Tathireddy P, Sharma R, Solzbacher F. Long-term bilayer encapsulation performance of atomic layer deposited $\mathrm{Al}_{2} \mathrm{O}_{3}$ and Parylene $\mathrm{C}$ for biomedical implantable devices. IEEE Transactions on Biomedical Engineering 2013;60(10):2943-2951

[8] Chen PJ, Rodger DC, Saati S, Humayun M, Tai YC. Microfabricated implantable parylenebased wireless passive intraocular pressure sensors. Journal of Microelectromechanical 
Systems 2008;17:1342-1351.

[9] Rodger DC, Fong AJ, Li W, et al. Flexible parylene-based multielectrode array technology for high-density neural stimulation and recording. Sensors and Actuators B 2008;132:449-460.

[10] Takeuchi S, Ziegler D, Yoshida Y, Mabuchi K, Suzuki T. Parylene flexible neural probes integrated with microfluidic channels. Lab on a Chip 2005;5:519-523.

[11] Tan CP, Cipriany BR, Lin DM, Craighead HG. Nanoscale resolution, multicomponent biomolecular arrays generated by aligned printing with parylene peel-off. NanoLetters 2010;10:719-725.

[12] Wright D, Rajalingam B, Karp JM, Selvarasah S, Ling Y, Yeh J, Langer R, Dokmeci MR, Khademhosseinil A. Reusable, reversibly sealable parylene membranes for cell and protein patterning. Journal of Biomedical Materials Research Part A 2008;85A:530-538.

[13] Chang TY, Yadav VG, De Leo S, Mohedas A, Rajalingam B, Chen C-L, Selvarasah S, Dokmeci MR and Khademhosseini A. Cell and protein compatibility of parylene-C surfaces. Langmuir 2007;23:11718-11725.

[14] Hoshino T, Saito I, Kometani R, Samejima K, Matsui S, Suzuki T, Mabuchi K, Kato YX. Improvement of neuronal cell adhesiveness on parylene with oxygen plasma treatment. Journal of Bioscience and Bioengineering 2011;113:395-398.

[15] Lu B, Lin J, Liu Z, Lee YK, Tai YC. Highly flexible, transparent and patternable parylene-C superhydrophobic films with high and low adhesion. Proc. IEEE 24th International Conference on MEMS, Cancun, Mexico 2011:1143-1146.

[16] Delivopoulos E, Murray AF and Curtis JC. Effects of parylene-C photooxidation on serumassisted glial and neuronal patterning. Journal of Biomedical Material Research 2010;94:47-58.

[17] Bursac N, Parker KK, Iravanian S, Tung L. Cardiomyocyte cultures with controlled macroscopic anisotropy - A model for functional electrophysiological studies of cardiac muscle. Circulation Research 2002;91(12):E45-54.

[18] McDevitt TC, Angello JC, Whitney ML, et al. In vitro generation of differentiated cardiac myofibers on micropatterned laminin surfaces Journal of Biomedical Materials Research 2002;60:472-479.

[19] Camelliti P, Gallagher JO, Kohl P, McCulloch AD. Micropatterned cell cultures on elastic membranes as an in vitro model of myocardium. Nature Protocols 2006;1(3):1379-1791. 
[20] Andersson H, van den Berg A. Where are the biologists? Lab Chip 2006;6:467-470.

[21] Rao $C$ et al. The effect of microgrooved culture substrates on calcium cycling of cardiac myocytes derived from human induced pluripotent stem cells. Biomaterials 2013;34:2399-2411.

[22] Lee JN, Jiang X, Ryan D, Whitesides GM. Compatibility of mammalian cells on surfaces of poly(dimethylsiloxane). Langmuir 2004;20(26):11684-11691.

[23] Michel R, Lussi JW, Csucs G, Reviakine I, Danuser G, Ketterer B, Hubbell JA, Textor M, Spencer ND. Selective molecular assembly patterning: a new approach to micro- and nanochemical patterning of surfaces for biological applications. Langmuir 2002;18:3281-3287.

[24] Fan CY, Tung Y-C, Takayama S, Meyhöfer E, Kurabayashi K. Electrically programmable surfaces for configurable patterning of cells. Advanced Materials 2008;20:1418-1423.

[25] Trantidou T, Prodromakis T, Toumazou C. Oxygen plasma induced hydrophilicity of Parylene-C thin films Journal of Applied Surface Science 2012;261:43-51.

[26] Brand NJ, Lara-Pezzi E, Rosenthal N, Barton PJ. Analysis of cardiac myocyte biology in transgenic mice: a protocol for preparation of neonatal mouse cardiac myocyte cultures Methods Molecular Biology 2010;633:113-124.

[27] Siedlecka U. Effects of clenbuterol on contractility and $\mathrm{Ca}^{2+}$ homeostasis of isolated rat ventricular myocytes. American Journal of Physiology - Heart and Circulatory Physiology 2008;295(5):H1917-26.

[28] Seong JW, Kim KW, Beag YW, Koh SK, Yoon KH, Lee JH. Effects of ion bombardment with reactive gas environment on adhesion of $\mathrm{Au}$ films to Parylene $\mathrm{C}$ film. Thin Solid Films 2005;476:386-390.

[29] Song JS, Lee S, Jung SH, Cha GC, Mun MS. Improved biocompatibility of parylene-C films prepared by chemical vapour deposition and the subsequent plasma treatment. Journal of Applied Polymer Science 2008;112:3677-3685.

[30] Walboomers XF, Monaghan W, Curtis ASG, Jansen JA. Attachment of fibroblasts on smooth and microgrooved polystyrene. Journal of Biomedical Materials Research 1999;46:212-220.

[31] Shattock MJ, Bers DM. Rat vs. rabbit ventricle: Ca flux and intracellular $\mathrm{Na}$ assessed by ionselective microelectrodes. American Journal of Physiology 1989;256:C813-C822.

[32] Venetucci LA, Trafford AW, Eisner DA. Increasing ryanodine receptor open probability alone does not produce arrhythmogenic calcium waves: threshold sarcoplasmic reticulum calcium 
content is required. Circulation Research, 2007;100:105-111.

[33] Carmeliet E. Intracellular $\mathrm{Ca}(2+)$ concentration and rate adaptation of the cardiac action potential. Cell Calcium 2004;35(6):557-573.

[34] Stokke MK, Hougen K, Sjaastad I, Louch WE, Briston SJ, Enger UH, Andersson KB, Christensen G, Eisner DA, Sejersted OM, Trafford AW. Reduced Serca2 abundance decreases the propensity for $\mathrm{Ca} 2+$ wave development in ventricular myocytes. Cardiovascular Research, 2010;86: 63-71.

[35] Bien H, Yin L, Entcheva E. Cardiac cell networks on elastic microgrooved scaffolds. IEEE Engineering in Medicine and Biology Magazine 2003;200322:108-112.

[36] Walsh KB, Parks GE. Changes in cardiac myocyte morphology alter the properties of voltagegated ion channels. Cardiovascualr Research, 2002;55:64-75.

[37] Pong T, Adams WJ, Bray MA, Feinberg AW, Sheehy SP, Werdich AA, Parker KK. Hierarchical architecture influences calcium dynamics in engineered cardiac muscle. Experimental Biology and Medicine (Maywood) 2011;236:366-373.

[38] Bray MA, Adams WJ, Geisse NA, Feinberg AW, Sheehy SP, Parker KK. Nuclear morphology and deformation in engineered cardiac myocytes and tissues. Biomaterials 2010;31:5143-5150.

[39] Geisse NA, Sheehy SP, Parker KK. Control of myocyte remodeling in vitro with engineered substrates. In Vitro Cellular and Developmental Biology- Animal, 2009;45:343-350.

[40] Parker KK, Tan J, Chen CS, Tung L. Myofibrillar architecture in engineered cardiac myocytes. Circulation Research 2008;103:340-342. 


\section{Appendix}
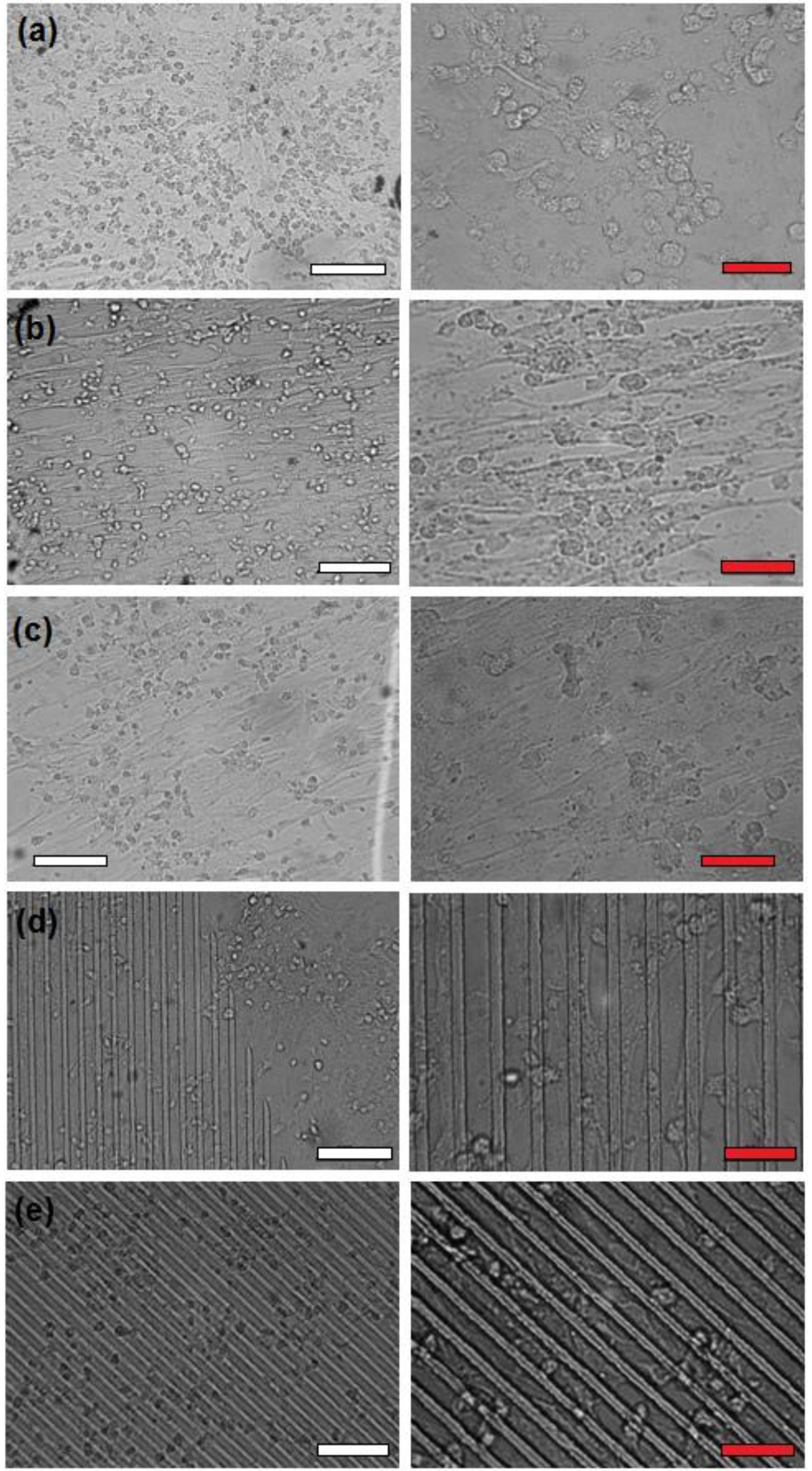

Figure A1. Bright Field microscopy images at x10 (left) and x40 magnification (right) of NRVM seeded: (a) on 
unpatterned constructs, on $\mathrm{H} / \mathrm{H}$ patterned constructs (b) 1 week and (c) 4 weeks after production, on $\mathrm{G} / \mathrm{H}$ patterned constructs (d) 1 week and (e) 4 weeks after production indicating the ability of the proposed technique to retain long-term patterning, scale bars: white $100 \mu \mathrm{m}$, red $50 \mu \mathrm{m}$. 
(a) $\mathrm{i}$

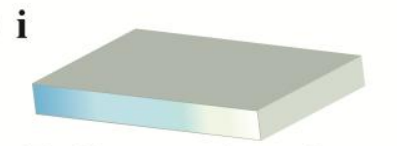

Glass or other substrate

ii

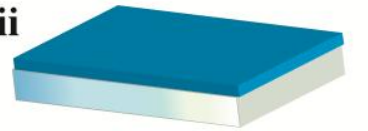

Parylene C

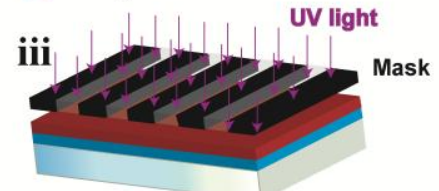

Lithography

$\square$ Photoresist

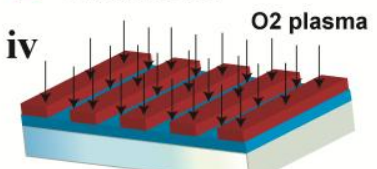

Oxygen plasma treatment

(d)

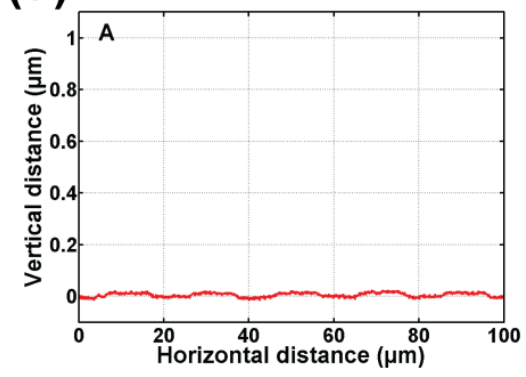

$\mathbf{v}$

V

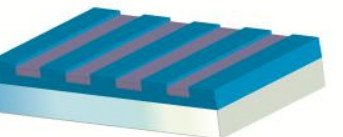

Hydrophobic Parylene $\square$ Hydrophilic Parylene

vi

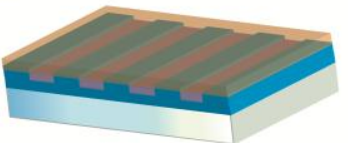

Attachment factors (fibronectin or collagen) vii

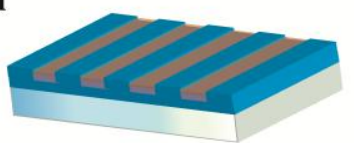

Attachment factors constrain on hydrophilic areas

viii

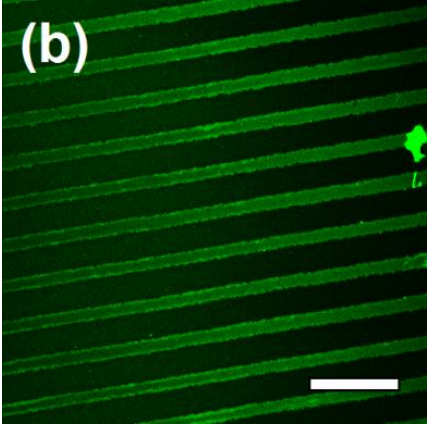

\section{(c)}

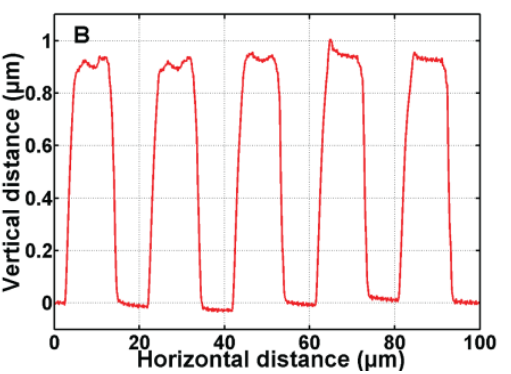

Figure 1. (a) Schematic representation of the fabrication process of the micro-engineered constructs. Immunofluorescence images of tagged fibronectin (green fluorescent) on (b) micro-engineered Parylene C constructs and (c) untreated (hydrophobic) Parylene C constructs, scale bar $50 \mu \mathrm{m}$. (d) Profilometer scan profiles of micro-engineered constructs: $\mathrm{A}-50 \mathrm{~W} / 1 \mathrm{~min}, \mathrm{~B}-400 \mathrm{~W} / 15 \mathrm{~min}, \mathrm{C}-400 \mathrm{~W} / 5 \mathrm{~min}$. 

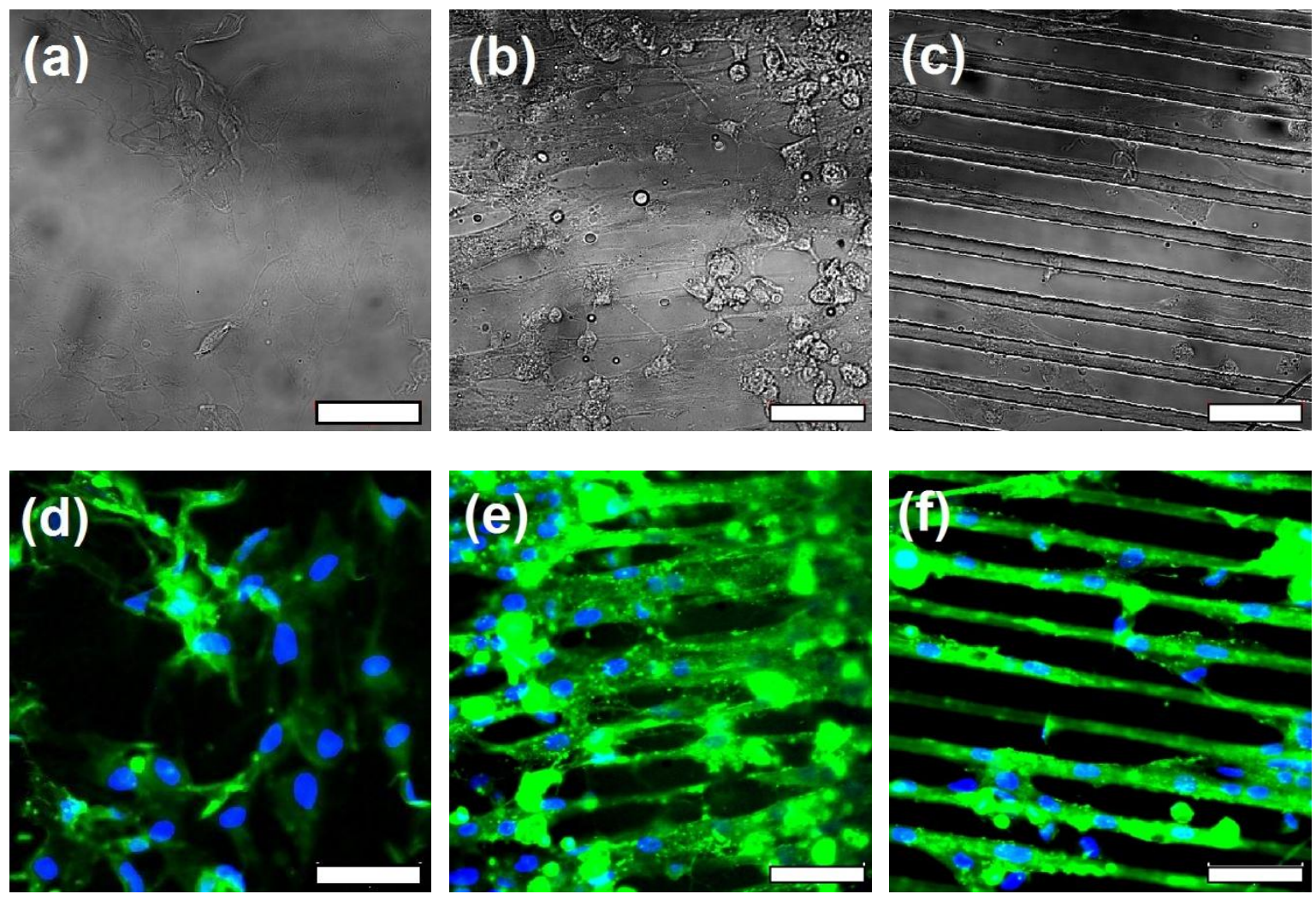

(g)

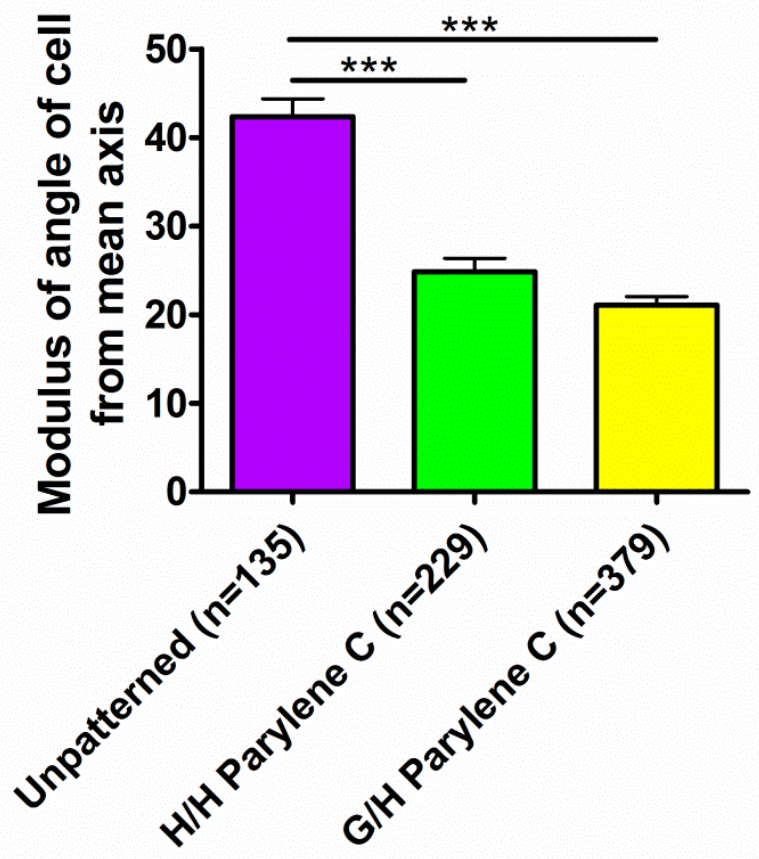

Figure 2. Bright field (top) and immunofluorescence (bottom) images of NRVM cultured on fibronectin

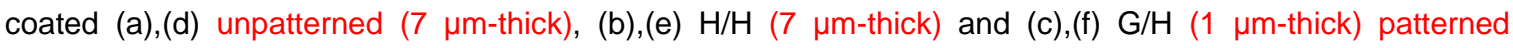
Parylene C constructs. Green -Myosin heavy chain antibody, Blue - DAPI, scale bar $50 \mu \mathrm{m}$. (g) Quantification of cell alignment on unpatterned and patterned constructs indicating the modulus of angle of cell from mean axis based on the long axis of the nucleus. ${ }^{* *} p<0.01,{ }^{* * *} p<0.001$. 


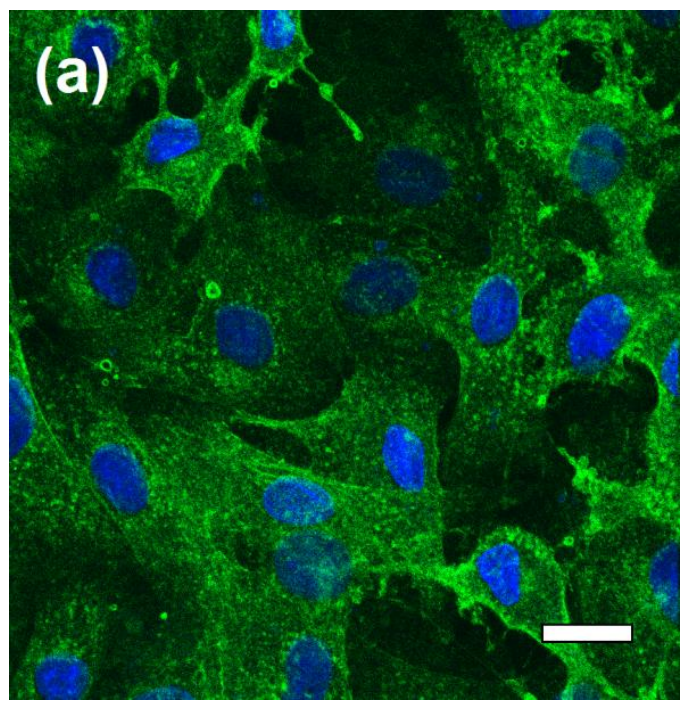

(c)

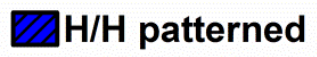

$\square$ Unpatterned

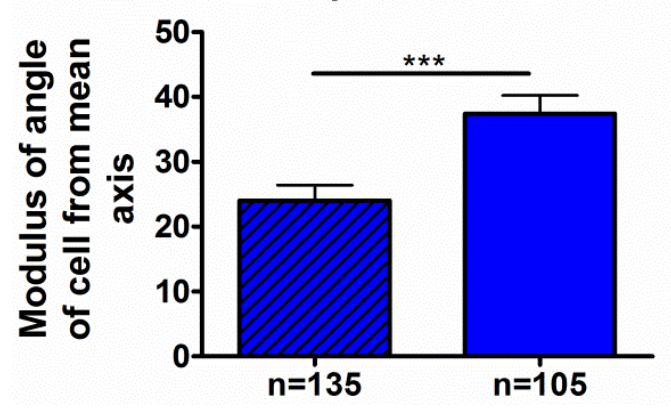

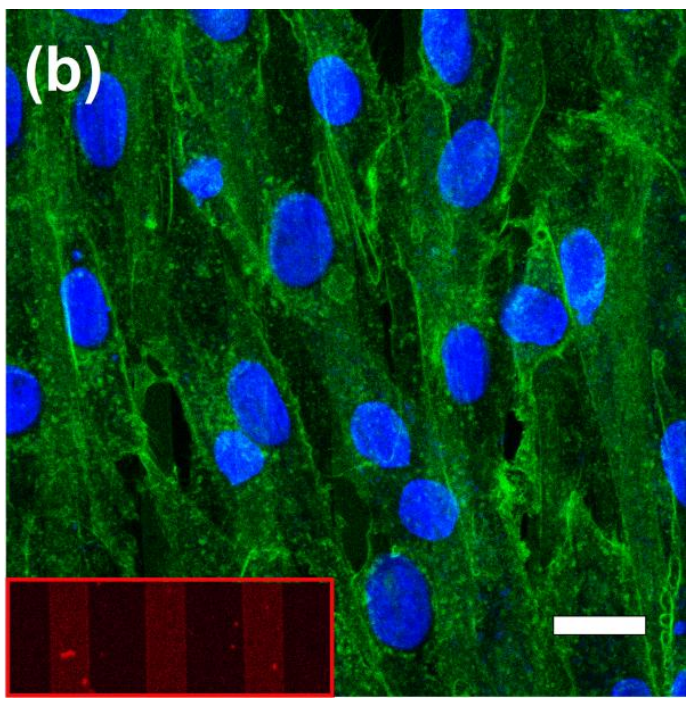

(d) $\square \mathrm{H} / \mathrm{H}$ patterned

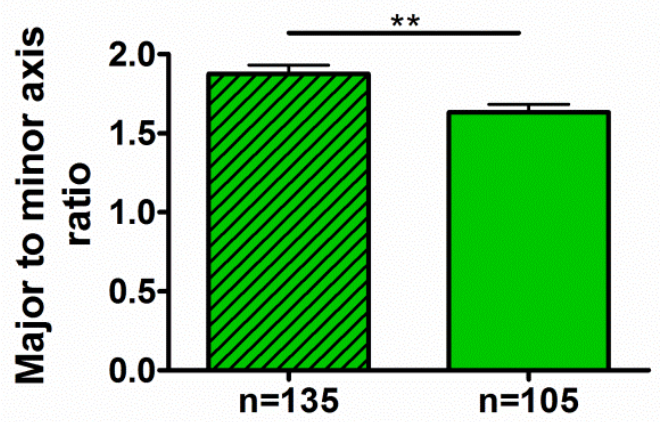

Figure 3. Immunofluorescence images of cardiac fibroblasts cultured on collagen coated (a) unpatterned and (b) patterned $10 \mu$ m-thick Parylene C constructs. Green -membrane stain di-8-ANEPPS, Blue -nuclear stain Hoechst 33258. (Inset) Collagen-coated micro-engineered constructs, scale bar $20 \mu \mathrm{m}$. (c) Modulus of angle of cell from mean axis of fibroblasts seeded on patterned and unpatterned constructs. (d) Major to minor cell axis ratio. ${ }^{* *} p<0.01,{ }^{* * *} p<0.001$. 

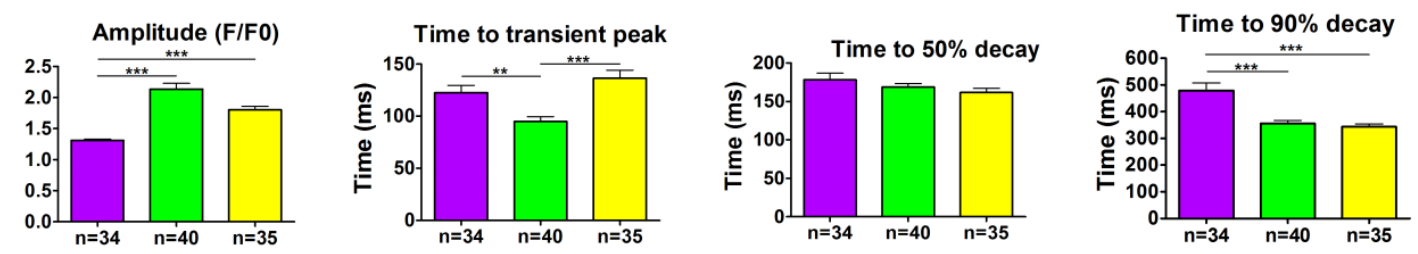

$1 \mathrm{~Hz}$ Field Stimulation
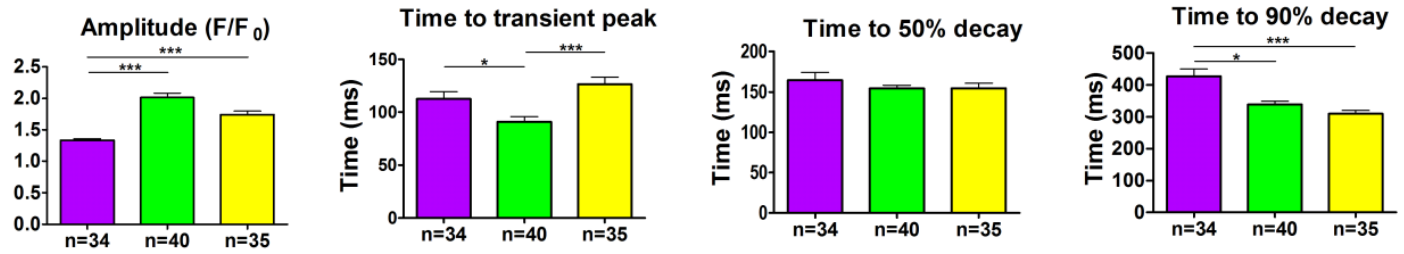

$2 \mathrm{~Hz}$ Field Stimulation
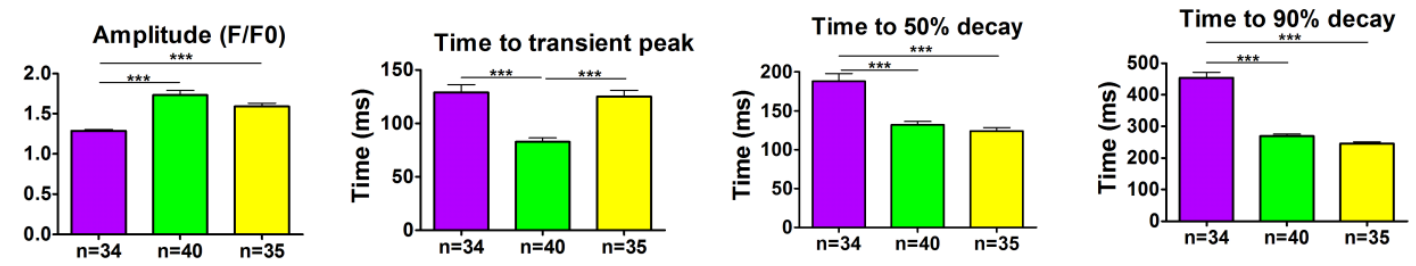

Unpatterned Parylene $C$

Hydrophilic/Hydrophobic Parylene C

Glass/Hydrophobic Parylene C

Figure 4. Fluorescent amplitude (f/f $\left.\mathrm{f}_{0}\right)$, time to peak (Tp), 50\% decay (T50), and $90 \%$ decay (T90) of the Ca $\mathrm{Ca}^{2+}$ transient of NRVM cultured on unpatterned, $\mathrm{H} / \mathrm{H}$ and $\mathrm{G} / \mathrm{H}$ patterned Parylene $\mathrm{C}$ constructs at $0.5 \mathrm{~Hz}, 1 \mathrm{~Hz}$ and $2 \mathrm{~Hz}$ field stimulation. ${ }^{*} \mathrm{p}<0.05,{ }^{* *} \mathrm{p}<0.01,{ }^{* * *} \mathrm{p}<0.001$. 
(a)

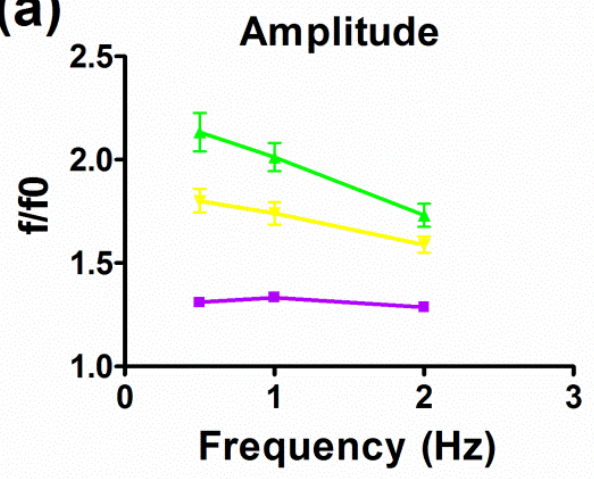

(c) Time to $50 \%$ decay

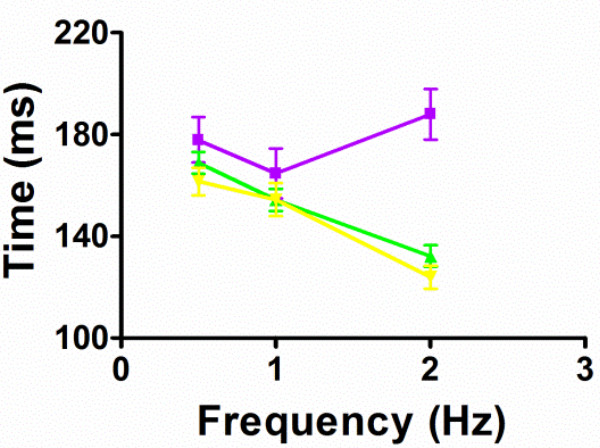

(b) Time to transient peak

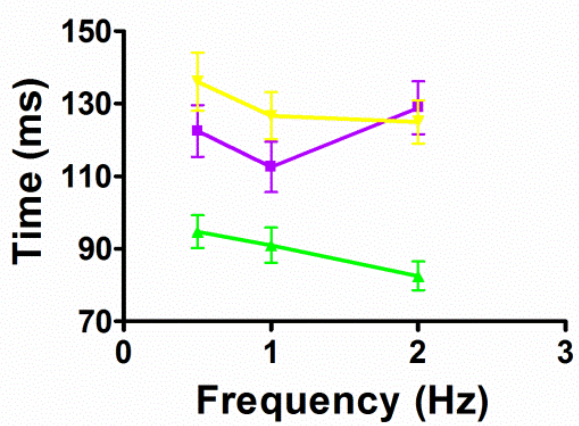

(d) Time to $90 \%$ decay

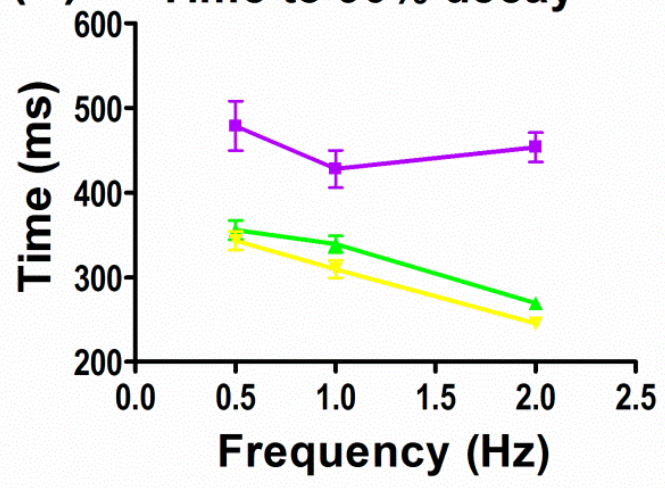

$\rightarrow$ Unpatterned Parylene C ( $n=34)$

- Hydrophilic/Hydrophobic Parylene C $(n=40)$

Glass/Hydrophobic Parylene C ( $n=35)$

Figure 5. Frequency dependency of (a) $\mathrm{Ca}^{2+}$ transient amplitude, (b) time to transient peak, (c) time to $50 \%$ decay and (d) time to $90 \%$ decay of NRVM cultured on unpatterned (purple), $\mathrm{H} / \mathrm{H}$ (green) and G/H (yellow) patterned constructs. 